\title{
Calculational Derivation of a Counter with Bounded Response Time
}

\author{
Joep L.W. Kessels \\ Philips Research Laboratories, NL-5600 JA Eindhoven, The Netherlands
}

\begin{abstract}
A counter is calculationally designed by applying a functional way of programming, in which a machine is conceived as a function from states to behaviours. In the derivation the ultimate program is obtained by applying a series of correctness-preserving transformations on an initial program, which serves as the functional specification.

The design is generic in that it describes counters with all possible periods. The response time as well as the power dissipation of all these counters are bounded by values that do not depend on the period.
\end{abstract}

Key words: Distributed systems, asynchronous circuits, handshake circuits, recursive designs, systolic designs, bounded response time, counters, transformational design.

\section{Introduction}

The purpose of this paper is twofold: to demonstrate a methodology for VLSI design and to present the design of a counter offering both bounded response time and bounded power dissipation. In theoretical computer science, sequential programs and distributed programs are generally studied separately. Sequential programs are considered as state transformers (see [3], [4] and [9]), whereas in most models of distributed programs the communication aspect predominates (see [6], [8] and [14]). In VLSI design, however, both aspects are significant and, therefore, there is a need for design methods encompassing both of them. In the section which follows we propose a design method and in the subsequent sections we derive a counter design by applying that method.

The counter design is recursive, i.e. a counter with a large enough period consists of an extension cell and a subcounter with a smaller period. Moreover, the design is distributed, i.e. both the exchange of information and the synchronization among the cells are achieved by communication only. The merit of a distributed design is that it allows the internal timing of each cell to be independent of its environment and, therefore, such designs are more liberal with respect to their realization than clocked designs (see [1] and [13]). The design is specified and programmed in a CSP-like language (see [7] and [8]).

In a distributed design, a cell accepts a communication only when it is ready for it. A recursive design is said to offer bounded response time if all permissible communications are accepted within a time bounded by a value that does not depend on the size of the design. For a specific class of designs, the similar notion of constant response time is formally defined in [15]. The counter design offers 
bounded response time and moreover it allows a realization offering bounded power dissipation as well (see [2]). The latter property is based on the fact that the subcounter operates at more or less half the rate of the compound counter. For that reason the design cannot directly be realized as a clocked circuit. In [12] two recursive counter designs are presented, one of which can be realized as a clocked circuit. An implementation of a similar design in delay-insensitive circuits is given in [5].

\section{Transformational Design}

For complex VLSI functions there is a large gap between specification and design. Usually, the specification language differs from the design language and, more significantly, programs implicitly embody all kinds of design decisions. In the design of complex systems it is therefore difficult to bridge the gap between specification and design in one step. Not only is the design step difficult, but it will also be difficult to prove the correctness of such designs afterwards. This holds particularly for distributed systems, since these systems are in general more difficult to analyse than clocked systems, which are by definition sequential automata having a global clock signal as an en abling condition for all transitions.

To avoid these problems, we derive programs transformationally. We start with an initial program, which serves as a functional specification. The initial program does not necessarily satisfy the required performance characteristics (it may even be non-executable). The ultimate program is then derived by applying a series of correctness-preserving transformations to the initial program and the ultimate program is therefore functionally correct by construction.

We use the term machine for objects that engage in events, whereas the term process is reserved for the behaviour of such objects. In general, the future behaviour of a machine depends on its history. Histories resulting in the same future behaviour are considered equivalent, and the equivalence classes thus obtained are called states. A machine can therefore be conceived as a tail recursive function from states to processes (future behaviours). This view leads to a functional way of programming, in which the program of a process consists of two parts: a function definition of the corresponding machine and an application of that function to the initial state. We distinguish external events and internal events. The external events of a process are its communications with its environment, whereas the internal events are either internal communications or merely state transitions. Internal events are relevant only for the design's performance characteristics such as speed and power dissipation. The functional behaviour of a process is obtained by abstracting from its internal events. For processes $P$ and $Q$, the formula $P \simeq Q$ means that $P$ and $Q$ are functionally the same, i.e. they may differ only in performance. This relation between processes induces a relation on machines with the same state space.

The design of the counter is obtained by applying a series of transformations on an initial program, which serves as the functional specification. Each of the transformations modifies the performance properties but leaves the functional 
behaviour invariant. We apply two kinds of transformations: refinements and decompositions. Refinements transform the state space, whereas decompositions increase the degree of concurrency.

The refinements are based on a property that is discussed below. Let machine $M$ have state space $S$ and machine $M^{\prime}$ state space $S^{\prime}$ and let function $g$ be a function from $S^{\prime}$ to $S$. We say that $M^{\prime}$ is an $g$-refinement of $M$ if $M^{\prime} \simeq(M \circ g)$, i.e $M^{\prime}$ is equivalent to the function composition of $M$ and $g$. Function $g$ is then called the abstraction function. Let $M$ be recursively defined by functional $F$, i.e. $M=F(M)$, and let function $f$ from $S$ to $S^{\prime}$ be a right inverse of $g$, i.e. $g \circ f=1_{S}$. Machine $M^{\prime}$ defined by

$$
M^{\prime} \triangleq F\left(M^{\prime} \circ f\right) \circ g
$$

is then an $g$-refinement of $M$, where function $f$ is called the refinement function. The proof is by induction: we prove that $F\left(M^{\prime} \circ f\right) \circ g \simeq F(M) \circ g$ under the assumption that $M^{\prime} \simeq M \circ g$. All operations that we will use in the construction of functionals respect the equivalence relation and therefore $M \simeq N \Rightarrow F(M) \simeq$ $F(N)$.

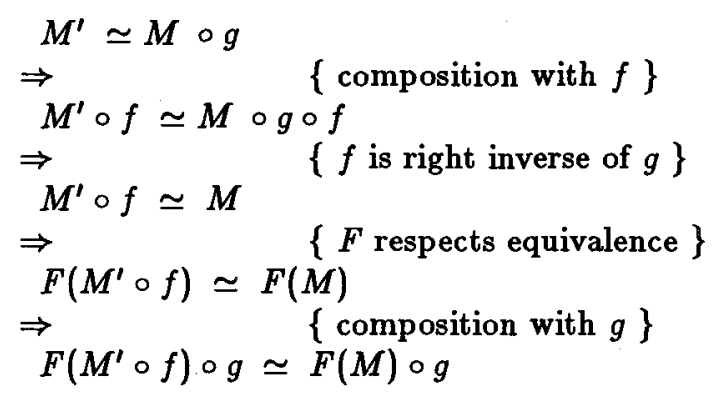

From the formula it follows that the definition of $M^{\prime}$ is obtained from the recursive definition of $M$ by replacing in the defining expression each occurrence of $M$ by $\left(M^{\prime} \circ f\right)$ and each occurrence of parameter $s$ by $g(s)$. The refinements in the derivation of the counter are special in that they are based on invertible abstraction functions which implies that the refinement functions are unique.

\section{Specification}

A counter is a machine with a binary port, say $A$, through which it outputs a periodic sequence of binary values. For positive $N$ we define $C_{N}$ as a counter with period $N$ and output sequence $\left(0^{N-1} 1\right)^{*}$. It is fairly straightforward to give an initial design in which machine $C_{N}$ has a natural number smaller than $N$ as its state space. In the programs that follow all parameters are natural numbers. Consider process

$$
\begin{aligned}
& C_{N}(0) \\
& \text { where } \\
& \quad C_{N}(n: n<N) \triangleq A !(n+1) \operatorname{div} N ; C_{N}((n+1) \bmod N)
\end{aligned}
$$


Process $C_{N}(n)$ outputs carry value $((n+1) \operatorname{div} N)$ and subsequently behaves as process $C_{N}((n+1) \bmod N)$. It is easy to verify that process $C_{N}(0)$, which is the behaviour of machine $C_{N}$ with initial state 0 , has the specified behaviour.

We can rewrite the program for $C_{N}$ by distinguishing the case when $n$ is smaller than $N-1$ from the case when $n$ equals $N-1$ (we leave out the domain restriction, which will only be given in the definition of a function).

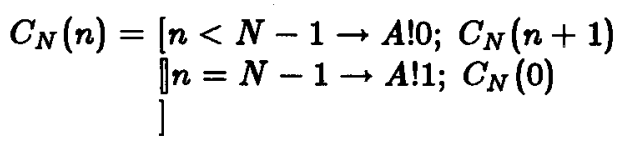

The design given above does not offer bounded response time since the arithmetic operations are performed on a variable $n$ whose range depends linearly on $N$. To obtain bounded response time we have to introduce concurrency. We therefore propose a recursive design consisting of a series of simple cells where each cell operates only on variables whose range is bounded by a value that does not depend on $N$. For such designs the response time between two external communications is defined as the number of ordered internal communications which have to be performed in between.

The recursive counter is shown in Fig.1. It consists of an extension cell $H$ and a subcounter $T$, which are connected by a binary channel $T$. $A$ through which the cell receives carry values from the subcounter. Cell $H$ therefore has two ports: an external port $A$ and an internal port T.A.

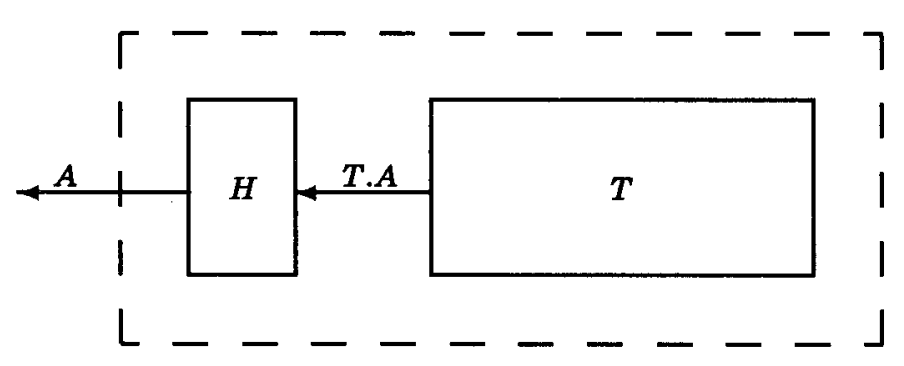

Fig. 1. Recursive design of counter

The subcounter has a shorter period than the compound counter. $C_{1}$ is the counter with the shortest period. We therefore propose $C_{1}$ as the tail cell, i.e. the counter without subcounter. The program for the tail cell follows directly from the general program given above, namely:

$$
C_{1}(0)=A ! 1 ; C_{1}(0)
$$




\section{First State Refinement}

For $N>1$ we must give a recursive decomposition of machine $C_{N}$. We aim at a decomposition in which the subcounter has period ( $N$ div 2$)$. We first derive a design for $N$ odd and we therefore write $N$ as $2 * M+1$ with $M>0$. For reasons of readability the subscript of $C_{N}$ is not rewritten.

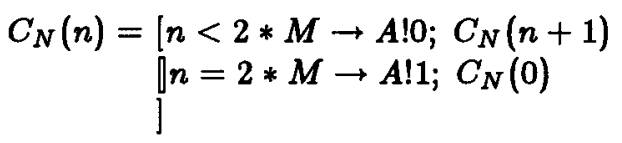

In the recursive design, each carry value of the subcounter must first be stored in the extension cell before it can be output. In the first refinement step, we therefore construct a machine $C_{N}^{(1)}$ in which the counter value is distributed over a binary carry value $c$ and a remainder $r$ smaller than $2 * M$.

In the sequel we will often partition a value $n$ into a quotient and a remainder with respect to a constant $N$ and therefore we define the function $P_{N}$

$$
P_{N}(n) \triangleq(n \operatorname{div} N, n \bmod N)
$$

We construct machine $C_{N}^{(1)}$ as a refinement of $C_{N}$ with refinement function $f_{N}$, where

$$
f_{N}(n: n<2 * M+1) \triangleq P_{2 * M}(n)
$$

The inverse abstraction function is

$$
f_{N}^{-1}(c, r)=2 * M * c+r
$$

with domain $(c<2) \wedge(r<2 * M) \wedge(c=0 \vee r=0)$.

The program of $C_{N}^{(1)}$ is obtained from the program of $C_{N}$ by the transformation described in an earlier section, i.e. by replacing in the program text of machine $C_{N}$ each occurrence of $C_{N}$ by $\left(C_{N}^{(i)} \circ f_{N}\right)$ and each occurrence of $n$ by $f_{N}^{-1}(c, r)$, so that

$$
\begin{aligned}
& C_{N}^{(1)}(c, r:(c<2) \wedge(r<2 * M) \wedge(c=0 \vee r=0)) \triangleq \\
& {\left[n<2 * M \rightarrow A ! 0 ; C_{N}^{(1)}\left(f_{N}(n+1)\right)\right)} \\
& \text { ln } n=2 * M \rightarrow A ! 1 ; C_{N}^{(1)}\left(f_{N}(0)\right) \\
& \text { where } n=f_{N}^{-1}(c, r)
\end{aligned}
$$

From the definition of $f_{N}$ follow two properties which can be used to simplify the program text of $C_{N}^{(1)}$ :

$$
\begin{gathered}
f_{N}^{-1}(c, r)<2 * M \quad \Leftrightarrow \quad c=0 \\
\text { and } \left.\left(f_{N}^{-1}(0, r)+1\right)\right)=P_{2 * M}(r+1)
\end{gathered}
$$

We can now rewrite the program text of $C_{N}^{(1)}$ in the following form: 


$$
\begin{gathered}
C_{N}^{(1)}(c, r)=\left[c=0 \rightarrow A ! 0 ; C_{N}^{(1)}\left(P_{2 * M}(r+1)\right)\right. \\
\| c=1 \rightarrow A ! 1 ; C_{N}^{(1)}(0,0)
\end{gathered}
$$

\section{Second State Refinement}

The parameter in the the subcounter must be smaller than $M$. In the second refinement step we therefore construct a machine $C_{N}^{(2)}$ in which the remainder of the counter value $r$ is split up into a binary value $a$ and a remainder $b$ smaller than $M$. In the subsequent decomposition we want to accommodate the binary values $a$ and $c$ in the extension cell and the value $b$ in the subcounter. There are many different ways in which the value of $r$ can be split into such values $a$ and $b$. If, however, we aim at a design with minimum power dissipation, we should minimize the number of communications between the extension cell and the subcounter. For that reason we take for $a$ the parity of $r$ and for $b$ the remaining value $(r \operatorname{div} 2)$. We construct machine $C_{N}^{(2)}$ as a refinement of $C_{N}^{(1)}$ with refinement function $g_{M}$, where

$$
g_{M}(c, r:(c<2) \wedge(r<2 * M) \wedge(c=0 \vee r=0) \triangleq(c, r \operatorname{div} 2, r \bmod 2)
$$

The abstraction function is

$$
g_{M}^{-1}(c, b, a)=(c, 2 * b+a)
$$

with domain $(c+a<2) \wedge(b<M) \wedge(c=0 \vee b=0)$.

The transformation is similar to the preceding one:

$$
\begin{gathered}
C_{N}^{(2)}(c, b, a:(c+a<2) \wedge(b<M) \wedge(c * b=0)) \triangleq \\
{\left[c=0 \rightarrow A ! 0 ; C_{N}^{(2)}\left(g_{M}\left(P_{2+M}(r+1)\right)\right) \quad \text { where } r=2 * b+a\right.} \\
\rrbracket c=1 \rightarrow A ! 1 ; C_{N}^{(2)}\left(g_{M}(0,0)\right) \\
]
\end{gathered}
$$

We rewrite the program text by distinguishing for $c=0$ the cases $a=0$ and $a=1$ :

$$
\begin{aligned}
C_{N}^{(2)}(c, b, a)= & {\left[c=0 \wedge a=0 \rightarrow A ! 0 ; C_{N}^{(2)}\left(g_{M}\left(P_{2 * M}(2 * b+1)\right)\right)\right.} \\
& \rrbracket c=0 \wedge a=1 \rightarrow A ! 0 ; C_{N}^{(2)}\left(g_{M}\left(P_{2 * M}(2 * b+2)\right)\right) \\
& \rrbracket c=1 \rightarrow A ! 1 ; C_{N}^{(2)}\left(g_{M}(0,0)\right) \\
] &
\end{aligned}
$$

To simplify the program text of $C_{N}^{(2)}$ we deduce two properties.

\section{Property 1:}

$$
\begin{array}{ll}
=g_{M}\left(P_{2 * M}(2 * b+1)\right) & \{b<M \Rightarrow 2 * b+1<2 * M\} \\
=g_{M}(0,2 * b+1) & \left\{\text { definition } g_{M}\right\}
\end{array}
$$




$$
(0, b, 1)
$$

\section{Property 2:}

$$
\begin{aligned}
& g_{M}\left(P_{2 * M}(2 * b+2)\right) \\
= & \left\{P_{2 * M}(2 * m)=(m \operatorname{div} M, 2 *(m \bmod M))\right\} \\
= & g_{M}((b+1) \operatorname{div} M, 2 *((b+1) \bmod M)) \\
& ((b+1) \operatorname{div} M,(b+1) \bmod M, 0)
\end{aligned}
$$

We rewrite the program text of $C_{N}^{(2)}$ by making two kinds of modifications. First, we substitute the definition of $g_{M}$ and simplify the program text using the properties given above. Secondly, we use domain restriction of $C_{N}^{(2)}$ to simplify the second guard.

$$
\begin{aligned}
C_{N}^{(2)}(c, b, a)= & {\left[c=0 \wedge a=0 \rightarrow A ! 0 ; C_{N}^{(2)}(0, b, 1)\right.} \\
& \llbracket a=1 \rightarrow A ! 0 ; C_{N}^{(2)}((b+1) \operatorname{div} M,(b+1) \bmod M, 0) \\
& \rrbracket c=1 \rightarrow A ! 1 ; C_{N}^{(2)}(0,0,0) \\
] &
\end{aligned}
$$

The third guard together with the domain restriction imply $(a=0 \wedge b=0)$ and therefore we can strengthen the guard to $(c=1 \wedge a=0)$ and replace in the corresponding command $C_{N}^{(2)}(0,0,0)$ by $C_{N}^{(2)}(0, b, 0)$. Subsequently, we can combine the first and the third guarded command and obtain the program given below:

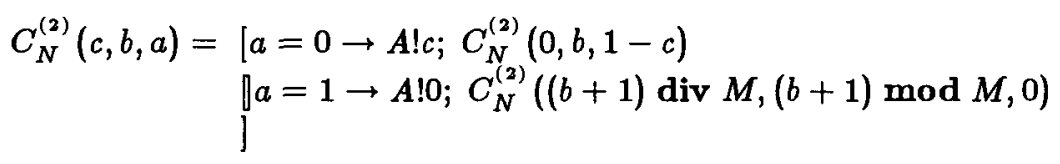

\section{Recursive Decomposition}

In the next design step, machine $C_{N}^{(2)}$ is decomposed into an extension cell $H_{1}$ containing the binary values $a$ and $c$, and a subcounter $C_{M}$ containing $b$. The definition of the decomposed design, denoted by $C_{N}^{(3)}$, is given below.

$$
C_{N}^{(3)}(c, b, a:(c+a<2) \wedge(b<M) \wedge(c * b=0)) \triangleq H_{1}(c, a) / / T: C_{M}(b)
$$

" $T: C_{M}$ " renames port $A$ of $C_{M}$ to $T . A$. Operator // denotes subordination (see [8]), i.e. concurrent composition with concealment of the internal communications (in this case T.A). Operator $/ /$ is asymmetric and, compared to the definition in [8], we have interchanged the operands.

The program for extension cell $H_{1}$ can be calculated from the programs for $C_{N}^{(2)}$ and $C_{N}^{(3)}$ : 


$$
\begin{aligned}
& C_{N}^{(3)}(c, b, a) \\
& \simeq\left\{\left(C_{N}^{(3)} \simeq C_{N}^{(2)}\right) \text { and definition of } C_{N}^{(2)}\right\} \\
& \mid a=0 \rightarrow A ! c ; C_{N}^{(3)}(0, b, 1-c) \\
& \llbracket a=1 \rightarrow A ! 0 ; C_{N}^{(3)}((b+1) \operatorname{div} M,(b+1) \bmod M, 0) \\
& =\left\{\text { definition of } C_{N}^{(3)}\right\} \\
& {\left[a=0 \rightarrow A ! c ;\left(H_{1}(0,1-c) / / T: C_{M}(b)\right)\right.} \\
& \llbracket a=1 \rightarrow A ! 0 ;\left(H_{1}((b+1) \operatorname{div} M, 0) / / T: C_{M}((b+1) \bmod M)\right) \\
& =\left\{A \text { is only a port of } H_{1}\right\} \\
& {\left[a=0 \rightarrow\left(A ! c ; H_{1}(0,1-c)\right) / / T: C_{M}(b)\right.} \\
& \rrbracket a=1 \rightarrow\left(A ! 0 ; H_{1}((b+1) \operatorname{div} M, 0)\right) / / T: C_{M}((b+1) \bmod M) \\
& \simeq\{/ / \text { permits the introduction of matching internal communications }\} \\
& {\left[a=0 \rightarrow\left(A ! c ; H_{1}(0,1-c)\right) / / T: C_{M}(b)\right.} \\
& \rrbracket a=1 \rightarrow\left(\left(A ! 0 \| T \cdot A ? c^{\prime}\right) ; H_{1}\left(c^{\prime}, 0\right)\right) / / \\
& T:\left(A !(b+1) \operatorname{div} M ; C_{M}((b+1) \bmod M)\right) \\
& \text { ] } \\
& =\left\{\text { specification: } C_{M}(b)=A !(b+1) \operatorname{div} M ; C_{M}((b+1) \bmod M)\right\} \\
& {\left[a=0 \rightarrow\left(A ! c ; H_{1}(0,1-c)\right) / / T: C_{M}(b)\right.} \\
& \rrbracket a=1 \rightarrow\left(\left(A ! 0 \| T . A ? c^{\prime}\right) ; H_{1}\left(c^{\prime}, 0\right)\right) / / T: C_{M}(b) \\
& =\{/ / \text { distributes over } \llbracket\} \\
& {\left[a=0 \rightarrow A ! c ; H_{1}(0,1-c)\right.} \\
& \llbracket a=1 \rightarrow\left(A ! 0 \| T . A ? c^{\prime}\right) ; H_{1}\left(c^{\prime}, 0\right) \\
& \text { ] } / / T: C_{M}(b)
\end{aligned}
$$

From this expression and the definition of $C_{N}^{(3)}$ we arrive at the following definition of extension cell $H_{1}$ :

$$
\begin{aligned}
H_{1}(c, a: c+a<2) \triangleq & {\left[a=0 \rightarrow A ! c ; H_{1}(0,1-c)\right.} \\
& \prod a=1 \rightarrow\left(A ! 0 \| T . A ? c^{\prime}\right) ; H_{1}\left(c^{\prime}, 0\right) \\
] &
\end{aligned}
$$




\section{General Solution}

Counters with an even period can be derived similarly with $f_{2 * M}$ as first refinement function, where

$$
f_{2 * M}(n: n<2 * M) \triangleq P_{2 * M}(n+1)
$$

This leads to extension cell $H_{0}$ given below.

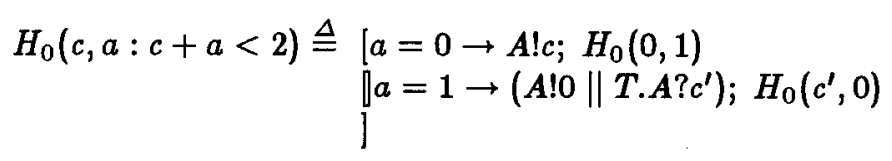

$H_{0}$ differs from $H_{1}$ in that, after producing a carry, it continues with $a=1$ instead of $a=0$. This stems from the fact that $f_{2 * M}(0)=(0,1)$ and the result is that in between two carries $H_{0}$ outputs one zero value less than $H_{1}$.

The total general refinement function $g f_{2 * M+x}$ with binary value $x$ is defined as the composition of the two refinement functions.

$$
g f_{2 * M+x}(n: n<2 * M+x) \triangleq g_{M} \circ f_{2 * M+x}
$$

where $f_{2 * M+x}(n)=P_{2 * M}(n+1-x)$. On the basis of this general refinement function we can construct a recursive design by replacing subcounters with a period larger than 1 by an abstraction of the compound counter:

$$
\begin{aligned}
& C_{2 * M+x}^{(4)}(n: n<2 * M+x) \triangleq\left[M=0 \rightarrow C_{1}(0)\right. \\
& \begin{array}{r}
\| M>0 \rightarrow H_{x}(c, a) / / T: C_{2 * M+x}^{(b)}(b) \\
\text { where }(c, b, a)=g f_{2 * M+x}(n)
\end{array}
\end{aligned}
$$

The parallel composition of the extension cell with the renamed subcounter and the subsequent concealment of the internal channels, denoted by " $H_{x}(c, a) / / T:$, can be thought of as a functional transforming counter process $C_{2 * M+x}^{(b)}(b)$ into counter process $C_{2 * M+x}^{(4)}(n)$.

Since the variables in the extension cells are bounded and each internal communication is performed concurrently with an external communication, the compound counter offers bounded response time if the subcounter offers bounded response time. By definition, tail cell $C_{1}$ offers bounded response time and thus we have shown by induction that $C_{N}^{(4)}$ offers bounded response time. The property of bounded response time is based on the fact that extension cell $H_{x}$ contains the least significant bit $a$ as well as the most significant bit $c$ of the counter value. In the design, least significant carries ripple from the extension cell to the subcounter (each communication is a least significant carry) and most significant carries ripple in the opposite direction (each output of the value one is a most significant carry).

Note that after concurrently performing an internal and an external communication $H_{x}$ performs at least one additional external communication. For that reason the average number of internal communications is at most half the 
number of external communications, which means that the subcounter operates maximally at half the speed of the compound counter. If the counter is implemented as an asynchronous CMOS circuit (see [1]), the resulting circuit will have an attractive power-dissipation property, since the power dissipation of such circuits depends linearly on the communication rate. The subcounter will therefore dissipate at most half the amount of the compound counter, which means that the power dissipation of the compound counter is at most twice the power dissipation of the extension cell. Hence, the counter not only offers bounded response time, but bounded power dissipation as well.

\section{Concluding Remarks}

We have transformationally derived the design of a recursive counter which means that the ultimate design is obtained by applying a series of correctnesspreserving transformations to an initial design, which served as the functional specification. Two kinds of transformations were applied: state refinements and parallel decompositions. Both kinds of transformations are based on simple axioms. All program transformations in the derivation of the counter are purely calculational and therefore are amenable to verifications by a theorem prover provided with the axioms mentioned before. The design of the counter is sufficiently simple to be obtained also by informal reasoning. More demanding examples are given in [10] and [11].

In the derivation, designs are described as functional programs in which machines are conceived as tail recursive functions from states to behaviours (processes). A program, therefore, consists of two parts: a function definition of the corresponding machine and an application of that function to the initial state. In the counter design, the function application is only given in the initial program, and in the transformation steps we only considered equivalent machines. In each transformation, the new initial state is obtained by applying the refinement function to the old initial state.

The design is distributed, i.e. it consists of a number of autonomous machines that proceed asynchronously and exchange information by explicit communication only. The state space is, therefore, strictly partitioned. In the counter design we show that a distributed program can be derived from a sequential one by decomposing the latter into a number of communicating subprograms. The state of the sequential program can then be thought of as the pseudo-global state of the distributed program. In distributed systems the internal timing of each subsystem can be independent of its environment In the design of the counter we exploit that fact by having the subcounter operate at most at half the rate of the compound counter. Therefore the power dissipation of the counter does not depend on the size of the counter.

The presented counter design is extreme in that each cell contains the minimum amount of information required for the solution. Such a solution is fast, but makes great demands on area, since it requires the maximum number of cells to construct a particular counter. If such a high speed is not required, a 
slower but cheaper design can be obtained by increasing the range of variable $a$ in each cell.

\section{Acknowledgements}

I thank Martin Rem and Ton Kalker for their critical and helpful comments. I am grateful to the other members of the VLSI Programming Club: Kees van Berkel, Ronald Saeijs, Frits Schalij and, in particular, Marly Roncken, who presented an alternative design.

\section{References}

1. van Berkel C.H., Kessels J.L.W., Roncken M.E., Saeijs R.W.J.J., and Schalij F.P.: The VLSI-programming language Tangram and its translation into handshake circuits. Proc. Eur. Conf. on Design Automation, Amsterdam (1991) 384-389

2. van Berkel C.H.: VLSI programming of a modulo-N counter with constant response time and constant power. Proc. Working Conference on Asynchronous Design Methodologies, Manchester (1992) (to appear)

3. Dijkstra E.W.: A Discipline of Programming. Series in Automatic Computation, Prentice-Hall International (1976)

4. Dijkstra E.W. and Scholten C.S.: Predicate Calculus and Program Semantics. Texts and Monographs in Computer Science, Springer Verlag (1989)

5. Ebergen J.C. and Peeters A.M.G.: Modulo-N counters: design and analysis of dealy insensitive circuits. Proc. 2nd Workshop on Designing Correct Circuits, Lyngby (1992) 27-46

6. Hennessy M.: Algebraic Theory of Processes. Series in the Foundation of Computing, The MIT Press Cambridge Massachusetts (1988)

7. Hoare C.A.R.: Communicating Sequential Processes. Comm. ACM 21 (1978) 666677

8. Hoare C.A.R.: Communicating Sequential Processes. Series in Computer Science, Prentice-Hall International (1985)

9. Hoare C.A.R. et al.: Laws of Programming. Comm. ACM 30 (1987) 672-686

10. Kessels J.L.W. and Rem M.: Designing Systolic, Distributed Buffers with Bounded Response Time. Distributed Computing 4 (1990) 37-43

11. Kessels J.L.W.: The Systematic Design of a Systolic RSA Converter. Proc. Workshop on Correct Hardware Design Methodologies, Turin (1991) Elsevier Science Publ., 235-251

12. Kessels J.L.W.: Designing Counters with Bounded Response Time. C.S. Scholten dedicata: van oude machines en nieuwe rekenwijzen, Academic Service, Schoonhoven The Netherlands (1991) 127-140

13. Martin A.J.: Compiling communicating processes into delay-insensitive VLSI circuits. Distributed Computing 1 (1986) 226-234

14. Milner R.: A Calculus of Communicating Systems. Lecture Notes in Computing Science 92 Springer-Verlag (1980)

15. Rem M.: Trace theory and systolic computations. Proc. Conf. Parallel Architectures and Languages Europe (Parle), Eindhoven, The Netherlands. Vol. 258, Lecture Notes in Computer Science 258 Springer-Verlag (1987) 14-33 